\title{
Erratum to: Total tumor volume predicts survival following liver resection in patients with hepatocellular carcinoma
}

\author{
Mu-xing $\mathrm{Li}^{1}$ • Hong Zhao ${ }^{1} \cdot \mathrm{Xin}-\mathrm{yu} \mathrm{Bi}^{1} \cdot \mathrm{Zhi}^{\mathrm{yu}} \mathrm{Li}^{1} \cdot \mathrm{Zhen} \mathrm{Huang}^{1} \cdot$ Yue Han $^{2}$ • \\ Jian-guo Zhou ${ }^{1}$ - Jian-jun Zhao ${ }^{1}$ Ye-fan Zhang ${ }^{1}$ - Wen-qiang Wei $^{3}$ • Dong-bin Zhao ${ }^{1}$. \\ Jian-qiang Cai ${ }^{1}$
}

Published online: 4 March 2016

(C) International Society of Oncology and BioMarkers (ISOBM) 2016

Erratum to: Tumor Biol.

DOI 10.1007/s13277-016-4794-7

The original version of this article contained a mistake.

The same figures were published twice in the online version namely, figure $4 \mathrm{E}$ and figure $4 \mathrm{~F}$.

The corrected Figure 4 image is shown in the next page.

The online version of the original article can be found at http://dx.doi.org/ 10.1007/s13277-016-4794-7.

Jian-qiang Cai

caijianqiang@cicams.ac.cn

1 Department of Abdominal Surgical Oncology, Cancer Hospital, Chinese Academy of Medical Sciences and Peking Union Medical College (CAMS and PUMC), No. 17, Panjiayuan Nanli,

Beijing 100021, People's Republic of China

2 Department of Interventional Therapies, Cancer Hospital, Chinese Academy of Medical Sciences and Peking Union Medical College (CAMS and PUMC), No. 17, Panjiayuan Nanli, Beijing 100021, People's Republic of China

3 Department of Cancer Epidemiology, Cancer Hospital, Chinese Academy of Medical Sciences and Peking Union Medical College (CAMS and PUMC), No. 17, Panjiayuan Nanli, Beijing 100021,

People's Republic of China 

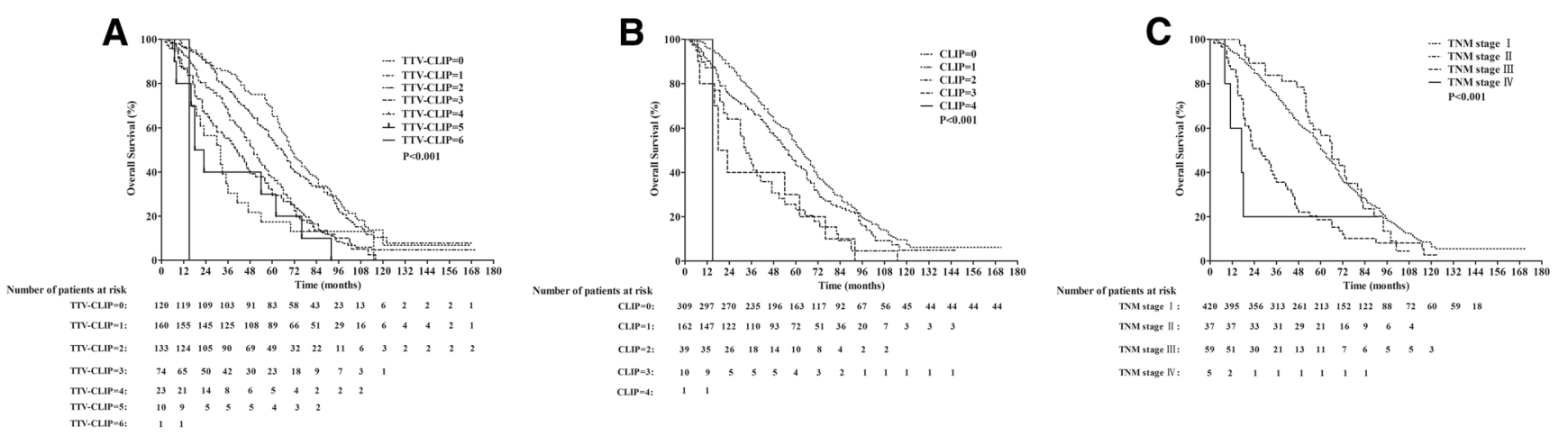

D

E
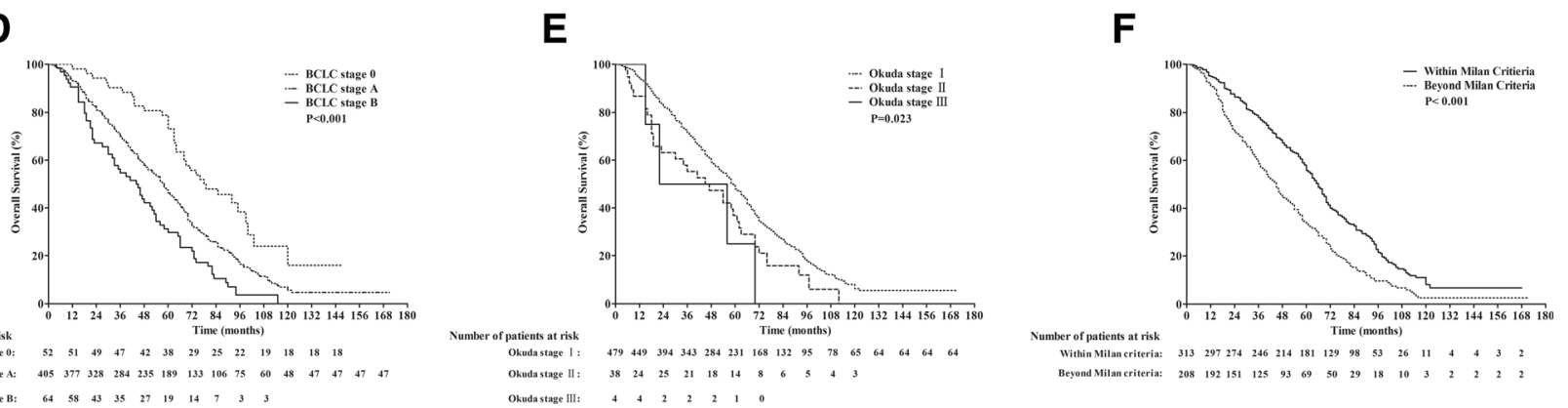

Fig. 4 Comparison of the survival distribution of the TTV-CLIP scoring system (a), CLIP scoring system (b), TNM staging system (c), BCLC staging system (d), Okuda staging system (e), and Milan criteria (f) 